\title{
Estándares para la realización de pruebas de función respiratoria. Procedimientos y recomendaciones
}

\author{
Standards for performing respiratory function tests. \\ Procedure and recommendations
}

Ireri Isadora Thirión Romero*

*Presidenta de la Sociedad Latinoamericana de Fisiología Respiratoria (SOLAFIRE).

\begin{abstract}
«...no nos hemos convertido en estatua: seguimos caminando, tropezando y cayendo, pero en marcha...»

Fernando Savater
\end{abstract}

Desde el año 2009 -en la revista Neumología y Cirugía de Tórax (NCT) - se han publicado los Procedimientos y Recomendaciones de las principales pruebas de función respiratoria (PFR). El propósito de esta serie de publicaciones es acercar el conocimiento técnico de la estandarización de las pruebas de función respiratoria a los lectores interesados y contribuir con ello a una mejor evaluación funcional respiratoria.

Gracias al apoyo de GlaxoSmithKline, de la Sociedad Mexicana de Neumología y Cirugía de Tórax y con la labor incansable de la Mesa Directiva de la Sociedad Latinoamericana de Fisiología Respiratoria (SOLAFIRE), hoy vemos reunidos -en forma de un Suplemento de Neumología y Cirugía de Tórax- los «Procedimientos y Recomendaciones».
En SOLAFIRE esperamos que este material contribuya, de forma práctica, con las actividades cotidianas de los cada vez más numerosos laboratorios de fisiología respiratoria que hay en Latinoamérica.

En SOLAFIRE queremos agradecer también a Neumología y Cirugía de Tórax su amable disposición para recopilar el material y publicarlo como un Suplemento. Nos complace ser testigos de cómo Neumología y Cirugía de Tórax mantiene sus puertas abiertas a nuevos proyectos.

Creemos que el Suplemento que usted tiene en sus manos es un estímulo para seguir difundiendo y generando conocimiento sin importar las fronteras. Dejemos atrás las celebraciones que perpetúan lo que nos distingue y comprendamos que compartimos algo más profundo e importante que lo que nos hace diversos.

iEnhorabuena!

Correspondencia:

Ireri Isadora Thirión Romero

Correo electrónico: draisadora.thirion@gmail.com 